Digital Press Social Sciences and Humanities

Harcèlement de rue à Paris dans le film Paris je t'aime sur la séquence de Quais de Seine

Nabila Nurul Putri and Suma Riella Rusdiarti

Proceeding of Conférence internationale sur le français 2018

Joesana Tjahjani, Merry Andriani, Sajarwa, Wening Udasmoro (eds) 


\title{
Harcèlement de rue à Paris dans le film Paris je t'aime sur la séquence de Quais de Seine
}

\author{
Nabila Nurul Putri* et Suma Riella Rusdiarti \\ Universitas Indonesia, Depok, Indonesia \\ *email : nabilanurul35@gmail.com
}

\section{Résumé}

Cet article montre la représentation d'harcèlement de rue qui est montrée dans la séquence « Quais de Seine » du film Paris, je t'aime. Le film dépeint l'histoire de femmes qui sont harcelées dans la rue par des étrangers qui l'appellent des mots désagréables basés sur la réalité, qui se produisent fréquemment à Paris. Harcèlement de rue est un harcèlement dans la rue, surtout sexuel qui se traduit par un harcèlement verbal et physique qui va souvent de pair. L'objectif de cette étude est de découvrir harcèlement de rue à Paris en se concentrant sur la séquence "Quais de Seine". La cinématographie et les éléments narratifs trouvés dans le film montrent des types de harcèlement, comment le harcèlement se produit aussi l'auteur et la victime de harcèlement. Les méthodes utilisées sont les études de cinéma structurel de Boggs, J.M., \& Petrie et la critique de genre de Simone de Beauvoir Cette recherche révèle que les victimes d'harcèlement de rue sont généralement des femmes de races diverses. Pendant ce temps, les auteurs sont des hommes de différentes races. De plus, cette recherche montre également que harcèlement de rue n'élève pas seulement l'aspect sexuel mais aussi l'aspect religieux.

\section{Mots-clés}

Corps, discrimination, film, harcèlement de rue, Paris

\begin{abstract}
This article talks about the depiction of harassment in the street happening in the Quais de Seine's scene in the film Paris Je t'aime. The film depicts the story of women harassed in the street by strangers who shouted to her uncomfortable words that usually happens in Paris. Street harassment is public bullying happening in the street, usually driven by sexual desire that transforms in to verbal and physical contacts. The goal of this research is to comprehend the street harassment focusing on Quais de Seine's scene in Paris Je t'aime. Cinematography and narrative elements show the type of harassment and how it is contested by both perpetrators and victims. This article uses qualitative and literary methods to analyze the data. The result shows that the victims of street harassment are generally experienced by women of color; whereas, the perpetrators are usually different men of color. Also, this research found that street harassment isn't only about sexual desire, but it also intersects with religious value.
\end{abstract}

\section{Keywords}

Film, sex, Paris, harassment

\section{Introduction}

Paris, la ville de l'amour et des amoureux, est la principale destination des touristes du monde. Chaque personne qui veut venir dans cette ville, imaginera les différentes scènes romantiques et belles qu'elles vont vivre. En réalité, Paris est une métropole aux multiples visages paradoxaux. Derrière son image de capitale de l'amour, ces dernières années, Paris souffre d'anxiété due à l'augmentation des cas 
d'harcèlement de rue, en particulier à l'encontre des femmes. Le harcèlement de rue, ce sont les comportements adressés aux personnes dans les espaces publics et semi-publics, visant à les interpeler verbalement ou non, leur envoyant des messages intimidants, insistants, irrespectueux, humiliants, menaçants, insultants en raison de leur sexe, de leur genre ou de leur orientation sexuelle (http://www.stopharcelementderue.org/quest-ce-que-le-harcelement-de-rue/). Les sifflements, les commentaires sexistes, les interpellations ou insultes, voire les attouchements, sont les comportements qui touchent les femmes et portent une atteinte inacceptable à leur dignité et à leur liberté. D'après une étude récente de l'Insee, 25\% des femmes âgées de 18 à 29 ans ont peur dans la rue, 20\% sont injuriées au moins une fois par an et $10 \%$ subissent des baisers ou des caresses qu'elles ne désirent pas. Et selon une étude réalisée par le Haut Conseil à l'égalité entre les femmes et les hommes, 100\% des femmes ont été harcelées au moins une fois dans les transports en commun et 76\% des Françaises ont été suivies au moins une fois dans la rue. Enfin une récente étude de la Fédération nationale des associations d'usagers des transports montre qu'à la question des éventuelles réactions des témoins, seuls $5 \%$ viennent en aide (Paris.fr, s. d.). On craint que ces chiffres augmentent.

Ce phénomène troublant de la ville de Paris a été soulevé au grand écran par plusieurs cinéastes au film Paris Je T'aime. Le film Paris Je T'aime est une combinaison de courts métrages d'environ de cinq minutes, révélant des problèmes sociaux dans la ville de l'amour, à Paris (Granec \& Menard, 2006, p. 2). La ville de Paris a toujours inspiré de nombreux cinéastes à créer leur travail dans le film. Ainsi, dans une des études sur le film de Paris Je T'aime de Myriam Thibault (2012) a expliqué que Paris est un lieu où tout peut arriver, y compris tous les dangers et tous les amours, car Paris est une ville qui ne dort jamais. Dans cette étude, la ville de Paris en tant que ville de rêve et idéaliste a exprimé de nombreux aspects tant positifs que négatifs. Bien que le thème principal porte sur l'amour, au film Paris Je T'aime a également présenté une histoire tragique et ironique, par exemple sur les séquences "La jeune fille au trench rouge" et "Quais de Seine".

La séquence "Quais de Seine" raconte un après-midi sur les rives de la Seine à Paris. Lorsque trois jeunes hommes étaient assis et passaient leur temps libre. Deux d'entre eux étaient occupés à flirter avec les femmes qui marchaient devant eux. Les femmes venaient d'ethnies différentes et la plupart d'entre elles ont été agressées verbalement pour leur apparence physique. L'une des femmes qui a attiré l'attention des jeunes était une fille maghrébine voilée, qui est tombée et a eu du mal à ranger son voile. Si d'autres femmes sont harcelées en raison de leur apparence et de leur apparence physique, cette fille maghrébine reçoit un traitement plus discriminatoire parce qu'elle est associée à Oussama Ben Laden. La prochaine partie du film montre que l'un des jeunes hommes présente ses excuses et essaie de se faire connaissance avec cette fille maghrébine. À travers ce synopsis, la séquence "Quais de Seine" montre de nombreux harcèlements de rue.

Cet article révélera harcèlement de rue dans le film Paris Je T'aime sur la séquence "Quais de Seine" en relation aux problèmes de genre et de discrimination. Les méthodes utilisées sont les études de cinéma structurel de Boggs, J.M., \& Petrie, D.W. (2008) dans son livre intitulé The Art of Watching Films. Les études de cinéma structurel commencent par explorer les éléments narratifs et cinématographiques du film, qui seront ensuite approfondis avec la compréhension du concept de harcèlement de rue, ainsi que la critique de genre de Simone de Beauvoir.

\section{Résultat et discussion}

\subsection{Epistémique}

\subsubsection{Harcèlement de rue : harcèlement corporel féminin}

Dans la séquence "Quais de Seine", il y a quatre personnages féminins qui deviennent des objets de harcèlement de rue. La première femme est blanche aux blonds, représente des groupes ethniques caucasiens. L'utilisation de gros plan extrême, met en évidence le sous-vêtement de femme qui semble sortir de son jeans à taille basse La caméra dans ce cas représente les yeux de jeunes gens qui font du corps féminin l'objet de son regard. 


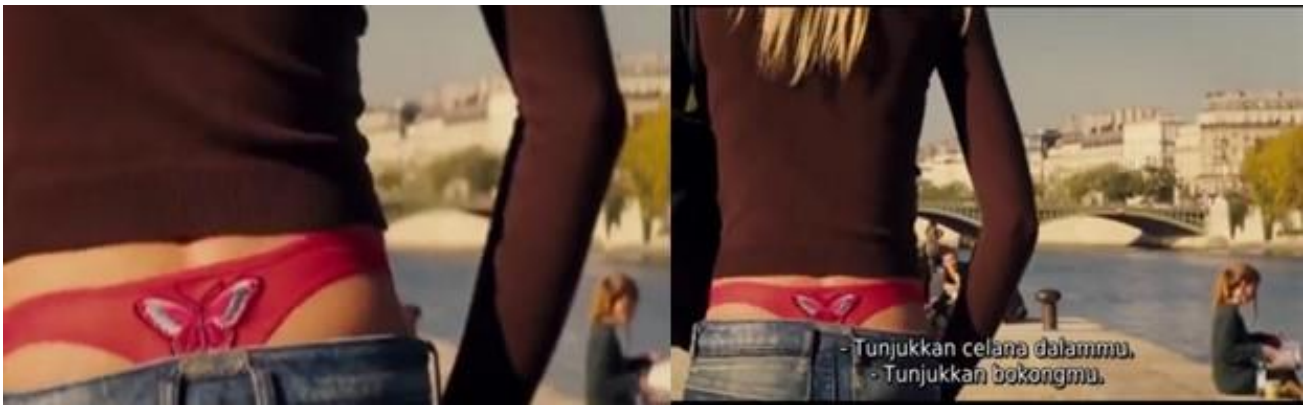

Figure 1 La gaze masculine dans le film (minutes 00: 08:03)

L'un des jeunes hommes a jeté les mots : "Fais voir la ficelle. Elle a un cul d'ouf». «Eh mademoiselle, tu me la files ta ficelle? Je n'ai plus de fil dentaire. Allez s'il te plait». Tous les mots qui exprimaient ce jeune homme est une forme de harcèlement sexuel verbal. Harcèlement de rue à la manière verbale ne nuit pas physiquement, mais psychologiquement, la victime se sentira perturbée, fâchée ou effrayée (Kearl, 2014, p. 8).

La deuxième femme victime d'abus dans le film est une femme asiatique. Avant que la figure féminine asiatique est montré, la caméra a capturé les expressions des jeunes hommes qui sont fascinés et disent "Oh!" À quelques secondes la caméra se déplace lentement du bas du corps de la fille et s'incline vers le haut de son corps, partant des jambes du corps, des cuisses, lentement jusqu'au visage. Une fois de plus, le mouvement de la caméra représentait le regard des hommes qui faisaient du corps féminin l'objet de son regard. Et ainsi de suite, des expressions verbales de la violence ont été entendues. « Mademoiselle, si je te paye un verre, je peux avoir un massage ? J'ai un bungalow juste à côté ». Ces déclarations verbales ont conduit à des actions physiques et à des préjugés ethniques. La femme asiatique présente dans le film, physiquement similaire aux femmes asiatiques de Thaïlande ou du Vietnam. Un salut verbal du «massage » affirme le stéréotype des femmes thaïlandaises ou vietnamiennes qui travaillent comme massothérapeutes. Cela montre que le harcèlement de rue peut également entraîner des sentiments de discrimination.

Les troisièmes femmes de victimes présentées sont deux femmes d'origine africaines noires. Dans cette scène, la prise de caméra utilisée est un plan long. Il n'y a pas l'accent sur une partie du corps ou sur un groupe ethnique, mais la violence verbale continue. «Bonjour mesdemoiselles, vous êtes charmantes à croquer aujourd'hui ». "Charmantes à croquer», ce ne sont en fait pas des mots vulgaires. C'est une sorte de compliment qui marque une certaine familiarité et une marque de plaisir qu'on ressent à la présence de l'autre. Mais ça peut laisser sous-entendre que l'homme en question trouve la femme attirante. Alors, lorsque cette expression a été faite dans un espace public à une personne que nous ne connaissions pas, cela pourrait causer de l'inconfort. Les femmes charmantes à croquer sont assimilées à des objets qui peuvent être appréciés pour satisfaire le désir sexuel.

Les femmes sont considérées comme des objets car l'un de leurs organes biologiques, le vagin, n'a pas de symbole sexuel fort comme un pénis (De Beauvoir, 1949, pp. 13-49). La subjectivité masculine est formée à partir des organes génitaux. Par conséquent, dans la société se forme une construction que les hommes en tant que sujets peuvent maîtriser tous les domaines. Par conséquent, les femmes ont le devoir d'obéir aux ordres des hommes et de satisfaire les désirs des hommes.

La quatrième femme victime dans le film est une femme maghrébine voilée noire. Au début, il semble que les jeunes hommes sur les rives de la Seine ne fassent pas attention ou ne fassent pas de la femme maghrébine un objet de leur regard, mais cette femme maghrébine reçoit également un harcèlement idéologique, ce qui s'expliquera dans la section suivante.

\subsection{Harcèlement de rue : Harcèlement idéologique contre une femme musulmane}

Les femmes maghrébines font partie intégrante de Paris en particulier et de la France en général. Bien que ce ne soit pas toujours le cas, les femmes maghrébines en France sont généralement attachées à leur identité de musulmanes. La figure de la femme musulmane dans la séquence "Quais de Seine » représentée par une fille qui s'appelle Zarqa. Comme les jeunes parisiens en général, Zarqa profite également de l'après-midi au bord de la Seine. Son apparence est typique d'une femme musulmane avec un voile sur la tête. Sa présence a été marquée par un regard d'un jeune homme que nous savons plus tard a été nommé François. François la regardait parce que Zarqa est tombée pour que son voile soit 
exposé par un vent fort. Elle s'est également blessée à la paume. François a sympathiquement essayé d'aider la fille.

À la différence des personnages féminins précédents, dans cette séquence, le personnage de Zarqa n'a pas accepté le harcèlement de deux jeunes hommes, l'ami de François. Cependant, ils dénoncent les bonnes actions de François en disant : «Si tu la touches c'est Oussama en personne, qui s'écrase dans ta face ».

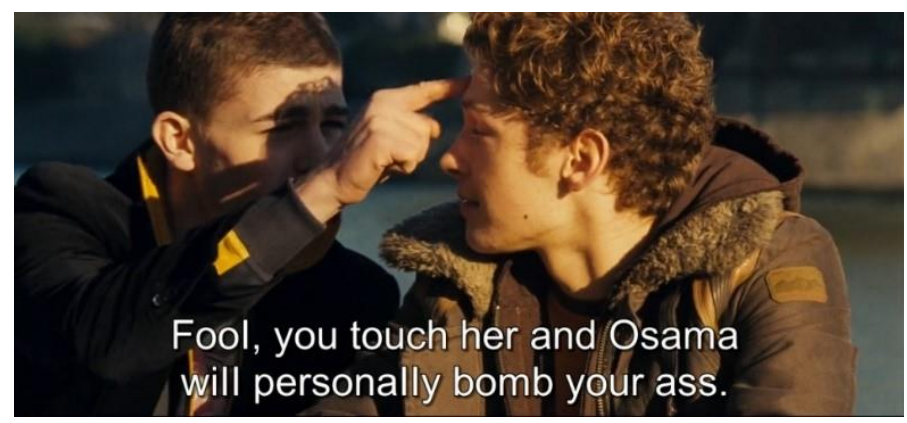

Figure 2 Zarqa et François parle du harcèlement aux femmes

Utiliser un gros plan, l'un des jeunes hommes a fait un mouvement en direction du front de François. Cela montre que l'homme a donné des idées ou une compréhension chez les autres (François). Ce gros plan a également le message important soit transmis au public que des femmes musulmanes ont des liens avec les terroristes. Le nom d'Oussama se réfère clairement à Oussama bin Laden. Le fondateur d'une organisation terroriste Al-Qaïda qui a perpétré l'un des plus importants attentats terroristes au monde, l'attentat à la bombe contre le World Trade Center et le Pentagone, le 11 septembre 2001. Relier directement les femmes musulmanes à Oussama peut ne pas être un harcèlement sexuel ou corporel, mais il vaut mieux l'appeler harcèlement idéologique. Cela peut aussi être lu comme une déclaration indirecte que les musulmans sont des terroristes. Une déclaration qui non seulement harcèle mais contient également des discriminations et des préjugés négatifs.

La présence de femme musulmane dans la séquence "Quais de Seine" prend le plus de temps par rapport aux autres femmes qui subissent du harcèlement sexuel. La figure de Zarqa en tant que femme maghrébine devient le point de vue du réalisateur pour montrer au public que la réalité du harcèlement de rue apporte aussi des abus sous forme de problèmes religieux. Le film met en évidence le stéréotype de l'islam en tant que religion dangereuse parce qu'il est lié à la relation du terrorisme. Le harcèlement religieux est une question plus sensible du harcèlement de rue.

La dernière scène du film montre également le personnage de François qui est décrit comme très sympathique et ne suit pas les déclarations de ses amis qui contiennent des préjugés contre les musulmanes. François a même gracieusement offert son aide à Susi pour apporter ses livres et accompagner Zarqa à la grande mosquée de Paris. La conversation entre eux a montré la curiosité de François à propos de la religion de l'islam et des musulmans. La scène de François et Zarqa contraste fortement avec la plupart des scènes remplies d'abus. Cette scène montre que les femmes et les hommes, tous deux étrangers dans un espace public, peuvent entamer une conversation ou une relation digne sans se rabaisser ou se harceler. Tout ce qui est nécessaire est une attitude sans préjugé et respect mutuel.

\section{Conclusion}

Selon l'analyse effectuée, cette étude révèle que les harcèlements de rue sont omniprésents et les jeunes femmes sont les premières victimes de ces comportements inacceptables dans l'espace public. Le harcèlement de rue peut être sous la forme des sifflements, des commentaires sexistes, des interpellations ou insultes, ou des attouchements. Les femmes deviennent des victimes, peu importe leur âge, leur apparence, leur race ou leur religion. Les femmes n'ont pas la liberté d'être humain ou de sujet libre. Elles restent des objets d'hommes.

L'analyse des figures révèle également que les hommes sont les principaux auteurs des abus. Cela confirme l'hypothèse selon laquelle les hommes se sentent libres d'attirer son attention et s'énervent quand les femmes ne répondent pas, que les femmes ne peuvent pas être aussi à l'aise dans l'espace 
public que les hommes. Le film montre aussi que les agresseurs sont des hommes d'origines raciales différentes.

Parmi les constats obtenus, harcèlement de rue dans la séquence "Quais de Seine" montre la situation réelle à Paris, en contraste aux attentes idéales de la société. Cela ouvre une nouvelle perspective sur la ville de Paris et le phénomène de harcèlement de rue. Les harcèlements dans la rue montrent également que cet aspect est très grand afin d'être étudié.

La scène finale montre le côté optimiste toujours offert par le film. Cela montre qu'il y a toujours un amour et une beauté à Paris. La conversation amicale entre François et Zarqa montre le côté de l'humanisme parisien que nous pouvons encore trouver et devenir le principal pour construire une société libre, égale et fraternelle dans la future.

\section{Références}

Boggs, J. M., \& Petrie, D. W. (2008). The Art of Watching Films (7th ed.). New York: McGraw Hill.

De Beauvoir, S. (1949). Le Deuxième Sexe (Vol. 2). Paris: Gallimard.

Kearl, H. (2014). Unsafe and Harrased in Public Spaces.

Laurence, G., \& Menard, K. (2006). Paris Je T'aime, Génération Amour. Cannes: La Fabrique de Film.

Paris.fr. (2016). Stop au harcèlement de rue. Retrieved from https://www.paris.fr/actualites/stop-auharcelement-de-rue-4276

Stop Harcèlement de Rue. (n.d.). Qu'est-ce que le harcèlement de rue ? Retrieved from http://www.stopharcelementderue.org/quest-ce-que-le-harcelement-de-rue/ 